\title{
Powered-descent landing GNC system design and flight results for Tianwen-1 mission
}

\author{
Xiangyu Huang ${ }^{1,2}(\bowtie)$, Chao $\mathbf{X u}^{1,2}$, Jinchang $\mathbf{H u}^{1,2}$, Maodeng $\mathbf{L i}^{1,2}$, Minwen Guo ${ }^{1,2}$, \\ Xiaolei Wang ${ }^{1}$, Yu Zhao ${ }^{1}$, Baocheng Hua ${ }^{1}$, and Yunpeng Wang ${ }^{1}$ \\ 1. Beijing Institute of Control Engineering, Beijing 100094, China \\ 2. National Key Laboratory of Science and Technology on Space Intelligent Control, Beijing 100094, China
}

\begin{tabular}{|c|c|}
\hline ABSTRACT & KEYWORDS \\
\hline The powered-descent landing (PDL) phase of the Tianwen- 1 mission began with composite & Tianwen-1 \\
\hline backshell-parachute (CBP) separation and ended with landing-rover touchdown. The & powered-descent landing (PDL) \\
\hline main tasks of this phase were to reduce the velocity of the lander, perform the avoidance & guidance, navigation, and contro \\
\hline maneuver, and guarantee a soft touchdown. The PDL phase overcame many challenges: & $(\mathrm{GNC})$ \\
\hline performing the divert maneuver to avoid collision with the CBP while simultaneously & backshell avoidance \\
\hline avoiding large-scale hazards; slowing the descent from approximately 95 to $0 \mathrm{~m} / \mathrm{s}$; per- & hazard avoidance \\
\hline forming the precise hazard-avoidance maneuver; and placing the lander gently and safely & \\
\hline on the surface of Mars. The architecture and algorithms of the guidance, navigation, and & Research Article \\
\hline control system for the PDL phase were designed; its execution resulted in Tianwen-1's & Received: 16 September 2021 \\
\hline successful touchdown in the morning of 15 May 2021. Consequently, the Tianwen-1 mission & Accepted: 23 October 2021 \\
\hline achieved a historic autonomous landing with simultaneous hazard and CBP avoidance. & (C) The Author(s) 2022 \\
\hline
\end{tabular}

\section{Introduction}

Tianwen-1 is China's first Mars exploration mission. The Tianwen-1 spacecraft consists of an orbiter and a landing rover (including the entry capsule and Zhurong Mars rover). It was launched on 23 July 2020, and the landing rover successfully landed on Mars on 15 May 2021.

The entry, descent, and landing (EDL) process was the most technically challenging phase of China's first Mars exploration mission. The Tianwen-1 EDL process has three phases: atmospheric entry, parachute descent, and powered-descent landing (PDL), as shown in Fig. 1. The PDL phase, which is the last major phase of the Tianwen-1 landing mission, starts with composite backshell-parachute (CBP) separation and ends with the detection of landing-rover touchdown. The guidance, navigation, and control (GNC) system plays a key role in achieving the Tianwen- 1 landing mission and faces its greatest challenges in the PDL phase.

The Tianwen-1 landing GNC system needs to be completely autonomous, mainly because of the communication time delay between the Earth and
Mars, which is approximately $38 \mathrm{~min}$. However, after atmospheric entry, the whole EDL process lasts only approximately $9 \mathrm{~min}$; thus, when people on the Earth obtain the first signal indicating atmospheric entry, landing is already concluded. Hence, the landing phase cannot be controlled with the ground in the loop and must be controlled completely autonomously. Regarding navigation for planetary PDL, inertial navigation or range-velocity-updated inertial navigation is the main method for determining the lander's poses in past planetary landing missions $[1,2]$. However, this method does not correct the lander's horizontal position. For improving the horizontal-position estimation of range-velocity-updated inertial navigation, image-aided navigation has been investigated for years [3, 4]. Not until the Perseverance Mars landing mission was terrain-relative navigation utilized in actual Mars landing flight for precision landing [5]. Regarding guidance for planetary PDL, researchers have investigated optimization guidance based on the pseudo-spectral method and a convex programming approach for Mars 


\section{Nomenclature}

$3 \mathrm{D}$

CBP

EDL

GNC three-dimensional composite backshell-parachute entry, descent, and landing guidance, navigation, and control

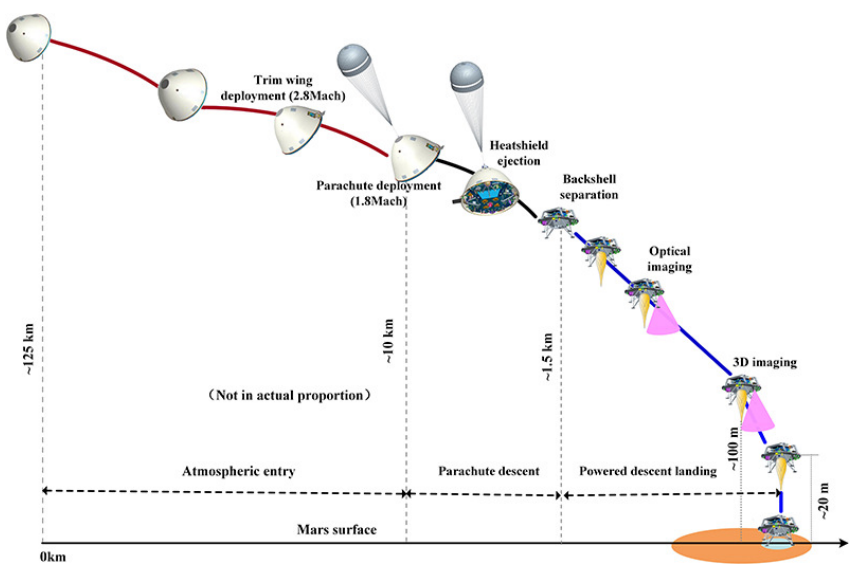

Fig. 1 EDL flight process of the Tianwen-1 mission. planetary PDL in the past Moon and Mars landing missions was polynomial guidance, which is based on the Apollo landing guidance algorithm [8].

The surface of Mars consists of various complex topographical features. Although the relatively flat area on the Utopian Plains of Mars was selected as the landing area for the Tianwen-1 mission, many craters, rocks, and humps exist in the landing area, which affect the landing safety. Therefore, an autonomous hazard recognition and avoidance capability is required for the Tianwen-1 PDL GNC system. Additionally, because the CBP jettisoned during the Mars EDL process will continue to fly, it may collide with the landing rover and may also cover the landing rover, endangering the rover's safety. Therefore, it is necessary to perform a divert maneuver to simultaneously avoid the CBP and hazards of the landing area in the PDL GNC system.

Furthermore, the uncertain initial velocity of the Mars PDL phase is significantly higher than that of the lunar hazard-avoidance phase, and faster deceleration during the PDL phase is needed owing to the constraints of the residual altitude and propellant availability. Therefore, the main task of the PDL GNC system is to use the main landing engine (MLE) to reduce the speed while autonomously performing CBP- and hazard-avoidance maneuvers.

\section{(10) UNIINRSITY PRESS}

$\begin{array}{ll}\text { M-HAS } & \text { multifunctional hazard-avoidance sensor } \\ \text { MLE } & \text { main landing engine } \\ \text { O-HAS } & \text { optical hazard-avoidance sensor } \\ \text { PDL } & \text { powered-descent landing }\end{array}$

In the previous Mars landing mission, the powered-descent phase of ExoMars 2016 was responsible for performing the backshell avoidance maneuver and deceleration [9]. The powered-descent phase of the Mars Science Laboratory and Mars 2020 was responsible for removing the final $0.1 \%$ of the kinetic energy; performing a 300-m out-of-plane divert maneuver to avoid collision with the backshell and parachute; and gently placing the rover on the surface using the skycrane maneuver [10, 11]. To date, only Chang'e-3 [12-14], Chang'e-4 [15], and Chang'e-5 [16] have achieved the autonomous hazard-avoidance function in the lunar landing mission, and Tianwen-1 first achieved the task of autonomous landing with simultaneous hazard and $\mathrm{CBP}$ avoidance in the Mars landing mission. The task is far more complex than lunar autonomous hazard avoidance and the powered descent in previous Mars landing missions.

For the aforementioned technical challenges and requirements of the Tianwen-1 PDL phase, this paper presents the resulting GNC system architecture and algorithm design, as well as the flight results.

\section{Overview of PDL phase}

For the main task and aforementioned challenges of the PDL GNC system, the PDL flight phase was designed (Fig. 1). In the PDL phase, the GNC system operates in the powered-deceleration mode, hover and imaging mode, hazard-avoidance maneuver mode, slow-descent mode, and no-control mode in turn according to the relay obstacle avoidance idea, which was derived in the Chang'e-3 landing mission [12-14]. The powered-deceleration mode begins with the CBP jettison and ends before the system enters the hover and imaging mode. The main task of this mode is to use the maximum thrust to reduce the landing rover's velocity, to avoid collisions with the CBP and to image and select a wide safe landing area for coarse hazard avoidance, in which the large-scale hazard areas that endanger landing safety are eliminated. This task provides a safe selection 
area for small-range precise hazard avoidance; avoids the risk that the field of view of the sensor contains hazards during low-altitude small-range three-dimensional (3D) imaging observations; and improves the safe landing probability of the system as a whole. In the hover and imaging mode, the landing rover maintains a hover state to capture 3D images of the landing area and then selects a more precise safe landing site. Once the landing site is selected, the GNC system switches to the hazard-avoidance maneuver mode to perform precise hazard avoidance and descent such that the landing rover descends to a $20-\mathrm{m}$ altitude above the landing site with a zero horizontal velocity and a preset value (approximately $1.5 \mathrm{~m} / \mathrm{s}$ ) of the vertical velocity. Next, the GNC system switches to the slow-descent mode, in which the landing rover slowly descends at a preset speed, reduces its horizontal velocity to zero, and maintains a vertical attitude. Once the landing rover's touchdown is detected, the GNC system sends a shutdown signal to turn off the MLE and then switches to the no-control mode, in which no orbit control or attitude control is performed.

\section{PDL GNC system description}

In this section, the PDL GNC system architecture and components are described. The PDL GNC system has standard components of a feedback control system (Fig. 2) with sensors, actuators, and computers, as well as GNC, hazard-identification, and safe landing site selection algorithms. The main objective of the PDL GNC system is to ensure that the terrain of the selected landing area, the final landing site, and the states (including the velocity, attitude angle, and attitude-angle rate) of touching the Mars surface are safe for the landing rover. The designed PDL GNC system consists of an inertial measurement unit (IMU), landing radars, hazard-avoidance sensors, 26 attitude-control thrusters, an MLE, and a GNC computer to perform the functions described in Section 2.

\subsection{Sensors}

\subsubsection{IMU}

A pair of IMUs are used, each of which consists of three orthogonal accelerometers and three orthogonal gyroscopes for measuring the specific acceleration and angular rate, respectively. The measuring range of the accelerometers is $-21 g$ to $21 g$, where $g=9.8 \mathrm{~m} / \mathrm{s}^{2}$. The measuring range of the gyroscopes is $-400\left(^{\circ}\right) / \mathrm{s}$ to 400 $\left({ }^{\circ}\right) /$ s. During the EDL, the IMUs are employed for inertial navigation and key event triggers.

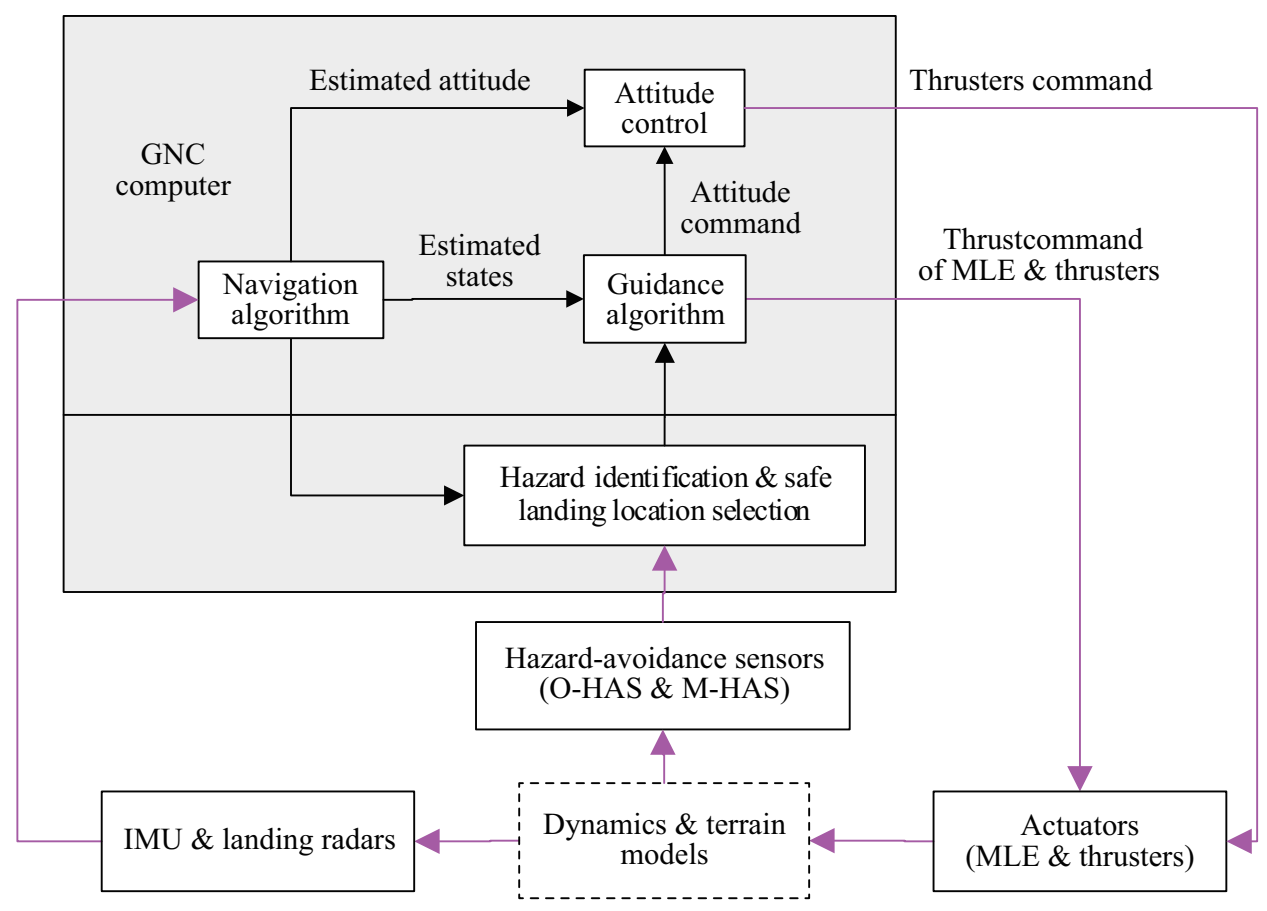

Fig. 2 GNC system architecture for the PDL phase. 


\subsubsection{Landing radars}

The landing radars consist of a suite of microwave radar and a suite of phased-array radar (PAR) for performing Mars-related measurements. The microwave radar contains four beams, each of which works automatically. The PAR contains nine beams, four of which are selected by the GNC system to provide measurements at every measurement time. Each beam for the two radars with a measuring range of 5-14 $\mathrm{km}$ can simultaneously measure the slant range and ground-relative velocity along its direction.

\subsubsection{Hazard-avoidance sensors}

Two hazard-avoidance sensors are equipped for hazard avoidance and landing-site selection. The first sensor, which is called the optical hazard-avoidance sensor (O-HAS), consists of a single optical imaging lens with a field of view of $30^{\circ}$. The second sensor, which is called the multifunction hazard-avoidance sensor (M-HAS), consists of an optical imaging lens and a laser imaging lens. The performance of the optical imaging lens of the M-HAS is identical to that of the O-HAS, and the laser imaging lens of the M-HAS also has a field of view of $30^{\circ}$. During the powered-deceleration mode, the O-HAS captures two-dimensional images of the landing area and then selects a safe landing area for coarse hazard avoidance. During the hover and imaging mode, the M-HAS collects 3D terrain data of the safe landing area and selects a safe landing site for precise hazard avoidance. An additional option for precise hazard avoidance is that the O-HAS can work in conjunction with the optical imaging mode of the M-HAS.

\subsection{Actuators}

$6 \times 25$-N thrusters and $20 \times 250$ - $\mathrm{N}$ thrusters are mounted for attitude control, of which $8 \times 250-\mathrm{N}$ thrusters are additionally utilized for translation control in the horizontal plane after the hover and imaging phase. Additionally, an MLE for translation control is employed to produce either a constant thrust of $7500 \mathrm{~N}$ or a throttleable thrust in the range of $1500-5000 \mathrm{~N}$.

\subsection{GNC computer}

The GNC computer collects and processes data from sensors, actuators, and onboard data-handling systems; performs real-time GNC calculations; and then sends control signals for orbit and attitude control. During the powered-descent phase, the GNC computer is also responsible for hazard identification and safe landing site selection using the images obtained from the hazard-avoidance sensors.

Figure 2 shows how the software programs of the GNC, hazard identification and safe landing, and location-selection algorithms run in the GNC computer. The general workflow of the PDL GNC system is described as follows. The navigation sensors measure and send the measurement data to the GNC computer. The navigation algorithm is responsible for processing the measurement data and sends the navigation results to the guidance algorithm and attitude-control algorithm. According to the navigation results and guidance parameters, the guidance algorithm forms the thrust command for the MLE and thrusters and sends the target attitude command to the attitude algorithm. The attitude algorithm forms the thruster command according to the navigation results and target attitude command. The GNC computer sends the thrust command to the MLE and attitude thrusters, which execute the command. During the hazard detection and avoidance process, the hazard-avoidance sensors send the surface image and terrain data of the landing area to the GNC computer, and the hazard identification and safe landing site selection algorithms process the surface image and $3 \mathrm{D}$ terrain data according to the navigation results to obtain the safe landing site information, which is sent to the guidance algorithms to complete the avoidance mission.

\section{PDL GNC algorithm}

\subsection{Guidance and control algorithm}

\subsubsection{Powered-descent deceleration guidance and control}

According to the dynamic characteristics of the CBP and entry platform (landing rover without the CBP and heatshield) and the simulation analysis of the flight trajectory of the separated CBP and entry platform, the following conclusions are drawn. (i) Considering the influence of the length of the rope between the parachute and the backshell, the avoidance distance of the CBP and entry platform must exceed $100 \mathrm{~m}$. (ii) If the horizontal velocity at the time of separation of the CBP and entry platform is $>15 \mathrm{~m} / \mathrm{s}$, the entry platform does not perform an avoidance maneuver, and the distance 
between the CBP and the entry platform satisfies the safe relative distance requirement. Considering the influence of the rough hazard-avoidance maneuver of the entry platform, the horizontal-velocity requirement for the no CBP avoidance maneuver is increased to $25 \mathrm{~m} / \mathrm{s}$. (iii) The CBP flight trajectory after separation is basically in the trajectory plane formed by the landing platform in the parachute descent process before separation.

According to the aforementioned conclusions, the strategy of the CBP- and hazard-avoidance maneuvers is detailed as follows (Fig. 3). If the horizontal velocity $v_{\text {hor-sep }}$ at the moment of separation of the CBP and entry platform is $>25 \mathrm{~m} / \mathrm{s}$, the $\mathrm{CBP}$ avoidance maneuver is not executed, and rough hazard-avoidance imaging and hazard-avoidance maneuvers are conducted when the entry platform reaches the preset altitude range (800-850 m). Otherwise, the CBP- and hazard-avoidance maneuvers are executed. The specific integrated determination strategy for the safe landing area of the CBP- and hazard-avoidance algorithm is described as follows.

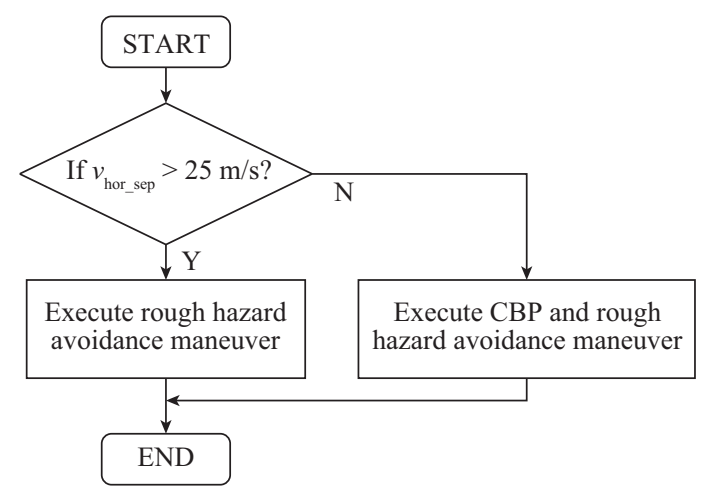

Fig. 3 Flowchart of the strategy for CBP- and hazardavoidance maneuvers.

In Fig. 4, the position of the entry platform is recorded and labeled as "A" when the entry platform height relative to the surface of Mars is $6 \mathrm{~km}$. The position of the entry platform is memorized and labeled as "B" after CBP separation, and the image of the landing area is simultaneously obtained using O-HAS. The trajectory plane is formed by the positions of $\mathrm{A}$ and $\mathrm{B}$ and the center location of Mars. The line of intersection of the trajectory plane with the local horizontal plane of Mars is A1_B1. The CBP after separation has no control and continues to fly at the velocity measured when it separates. Therefore, the CBP flight trajectory after separation is basically in
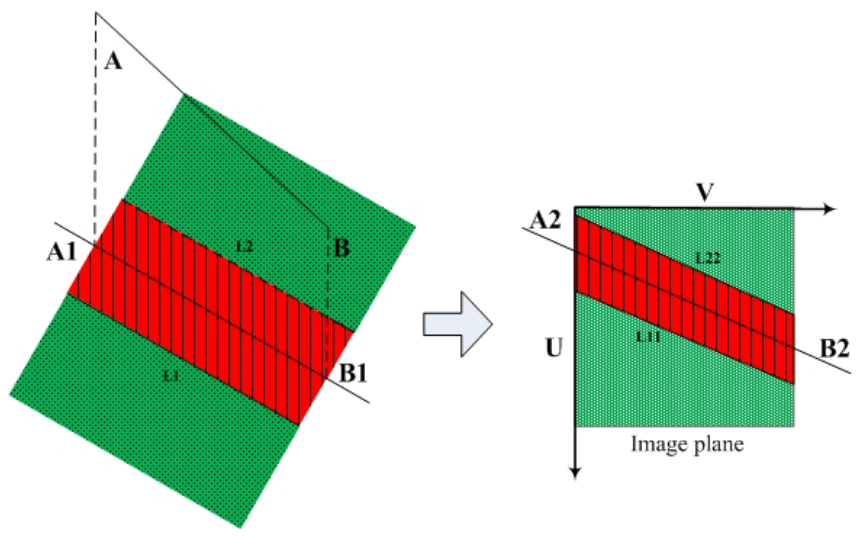

Fig. 4 Sketch of safe-area determination for hazard and CBP avoidance.

the trajectory plane, and the $\mathrm{CBP}$ lands in the area near Line A1_B1.

According to the requirements for the relative distance of CBP avoidance, the entry platform should fly away from the plane formed by Lines $\mathrm{A}_{-} \mathrm{B}$ and $\mathrm{A} 1_{-} \mathrm{B} 1$ as soon as possible, and the minimum flying distance is $200 \mathrm{~m}$ (distance from Line L1 or Line L2 to Line A1 B1); that is, the area between Lines L1 and L2 is a dangerous area to be avoided for the entry platform. The safe landing site selected for hazard avoidance should be outside Lines L1 and L2 (the minimum distance from Line A1 B1 is $200 \mathrm{~m}$ ). Considering that hazard recognition is realized using the optical image of the landing area, it is necessary to project Line A1_B1 onto the image plane to identify the dangerous area in the image plane. Next, the safe landing site is selected in the safe area (outside Lines L11 and L22) to simultaneously realize hazard avoidance and CBP avoidance.

To simultaneously satisfy the requirements of rapid deceleration and trajectory maneuvers, hazard-avoidance polynomial guidance is designed. The combination of the main engine with a maximum thrust of $7500 \mathrm{~N}$ and four 250-N thrusters is adopted. Once the thrust command given by the guidance algorithm is $<4500$ $\mathrm{N}$, the main-engine variable thrust control is switched on, and the four 250-N thrusters are shut down. If a safe landing site is not found, the current position on Mars under the entry platform is selected as the landing site. Once the safe landing site is obtained, 3D control of the position and velocity is performed so that hazard avoidance and/or CBP avoidance are realized are realized. The expression for the guidance command acceleration is

$$
\overline{\boldsymbol{a}}_{\mathrm{G}}=\overline{\boldsymbol{a}}_{\mathrm{tG}}-6\left(\overline{\boldsymbol{v}}_{\mathrm{tG}}+\overline{\boldsymbol{v}}_{\mathrm{G}}\right) / t_{\mathrm{go}}+12\left(\overline{\boldsymbol{r}}_{\mathrm{tG}}-\overline{\boldsymbol{r}}_{\mathrm{G}}\right) / t_{\mathrm{go}}^{2}
$$


where $\overline{\boldsymbol{a}}_{\mathrm{tG}}, \overline{\boldsymbol{v}}_{\mathrm{tG}}$, and $\overline{\boldsymbol{r}}_{\mathrm{tG}}$ represent the target acceleration, target velocity, and target position, respectively; $\overline{\boldsymbol{v}}_{\mathrm{G}}$ and $\overline{\boldsymbol{r}}_{\mathrm{G}}$ represent the current velocity and current position, respectively; and $t_{\text {go }}$ represents the to-go time for guidance, which is computed as

$$
t_{\mathrm{go}}= \begin{cases}\frac{2 v_{\mathrm{f}}+v}{a_{\mathrm{f}}}+\sqrt{\left(\frac{2 v_{\mathrm{f}}+v}{a_{\mathrm{f}}}\right)^{2}+\frac{6\left(r-r_{\mathrm{f}}\right)}{a_{\mathrm{f}}},} & a_{\mathrm{f}} \neq 0 \\ \frac{3\left(r_{\mathrm{f}}-r\right)}{v+2 v_{\mathrm{f}}}, & a_{\mathrm{f}}=0\end{cases}
$$

Here, $a_{\mathrm{f}}, v_{\mathrm{f}}$, and $r_{\mathrm{f}}$ are the vertical components of the target acceleration, velocity, and position, respectively; and $v$ and $r$ are the vertical components of the current velocity and position, respectively.

\subsubsection{Descent landing guidance and control}

Descent landing guidance consists of vertical and horizontal channels for the hover and imaging, hazardavoidance maneuver, and slow-descent modes.

(1) Vertical channel guidance

Vertical channel guidance involves continual trajectory control using the main engine with a variable thrust. The vertical channel guidance command acceleration is defined by the proportional-integral-derivative (PID) control of the altitude, vertical velocity, and vertical acceleration error, which is inherited from the Chang'e-3 landing mission [12-14]. The altitude, vertical velocity, and vertical acceleration of the reference trajectory are denoted as $h_{\mathrm{t}}, \dot{h}_{\mathrm{t}}$, and $\ddot{h}_{\mathrm{t}}$, respectively. The altitude, vertical velocity, and vertical acceleration of the current states provided by the navigation algorithm are denoted as $h, \dot{h}$, and $\ddot{h}$, respectively, where $\ddot{h}=a_{x}-g_{\mathrm{m}}, a_{x}$ represents the vertical thrust acceleration of the entry platform provided by navigation, and $g_{\mathrm{m}}$ represents the magnitude of the Mars' gravitational acceleration.

For the hover and imaging phase, the vertical command acceleration is defined as

$$
\begin{aligned}
\boldsymbol{a}_{\mathrm{cmd}, \mathrm{ver}}= & {\left[-c_{1} \cdot\left(h-h_{\mathrm{t}}\right)-c_{2} \cdot\left(\dot{h}-\dot{h}_{\mathrm{t}}\right)-\right.} \\
& \left.c_{3} \cdot\left(\ddot{h}-\ddot{h}_{\mathrm{t}}\right)+g_{\mathrm{m}}\right] \frac{\boldsymbol{r}}{\|\boldsymbol{r}\|}
\end{aligned}
$$

where $c_{1}, c_{2}$, and $c_{3}$ are the PID control coefficients for the altitude, vertical velocity, and vertical acceleration errors, respectively, and $\boldsymbol{r}$ is the current position vector of the entry platform provided by navigation. $c_{1}, c_{2}$, and $c_{3}$ are designed to be $0.08,0.6$, and 0.01 , respectively. The target parameters $h_{\mathrm{t}}, \dot{h}_{\mathrm{t}}$, and $\ddot{h}_{\mathrm{t}}$ are designed to be 100 $\mathrm{m}$ (expected hovering altitude), 0 , and 0 , respectively.
For the hazard-avoidance maneuver phase, the vertical command acceleration vector is defined as

$\boldsymbol{a}_{\mathrm{cmd}, \mathrm{ver}}=\left[-c_{1} \cdot\left(h-h_{\mathrm{t}}\right)-c_{2} \cdot\left(\dot{h}-\dot{h}_{\mathrm{t}}\right)+\ddot{h}_{\mathrm{t}}+g_{\mathrm{m}}\right] \frac{\boldsymbol{r}}{\|\boldsymbol{r}\|}$

where $h_{\mathrm{t}}, \dot{h}_{\mathrm{t}}$, and $\ddot{h}_{\mathrm{t}}$ are calculated according to the reference trajectory of the hazard-avoidance maneuver phase, which is initially designed as a uniform acceleration process followed by a uniform deceleration process with the main-engine minimum-thrust constraint (Fig. 5).

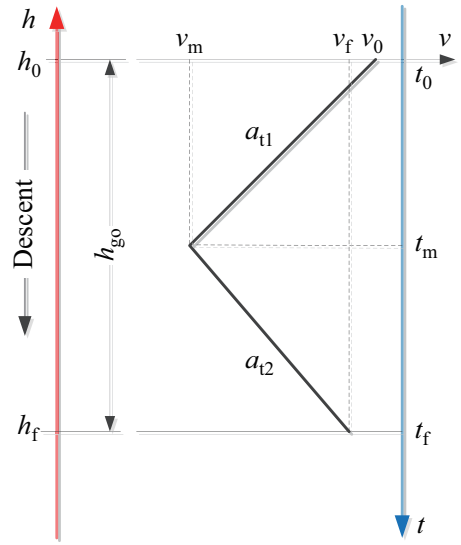

Fig. 5 Planned reference trajectory of the hazard-avoidance maneuver phase.

If the minimum thrust of the main engine is denoted as $F_{\text {min }}$ and the mass of the entry platform is denoted as $m_{0}$, the maximum descent acceleration can be calculated as follows:

$$
a_{\mathrm{dmax}}=\left(m_{0} \cdot g_{\mathrm{m}}-F_{\min }\right) / m_{0}
$$

Denoting the maximum time of the hazard-avoidance horizontal maneuver as $t_{\max }$, the available descent distance as $h_{\mathrm{go}}$, the uniform acceleration as $a_{\mathrm{t} 1}$, the uniform deceleration as $a_{\mathrm{t} 2}$, and the maximum descent velocity as $v_{\mathrm{m}}$, we obtain

$$
\left\{\begin{array}{l}
\frac{v_{\mathrm{m}}-v_{0}}{a_{\mathrm{t} 1}}+\frac{v_{\mathrm{f}}-v_{\mathrm{m}}}{a_{\mathrm{t} 2}}=t_{\mathrm{max}}=t_{\mathrm{f}}-t_{0} \\
\frac{v_{\mathrm{m}}^{2}-v_{0}^{2}}{2 a_{\mathrm{t} 1}}+\frac{v_{\mathrm{f}}^{2}-v_{\mathrm{m}}^{2}}{2 a_{\mathrm{t} 2}}=h_{\mathrm{go}}=h_{\mathrm{f}}-h_{0}
\end{array}\right.
$$

where $t_{0}, v_{0}$, and $h_{0}$ represent the initial time, initial vertical velocity, and initial altitude, respectively, of the hazard-avoidance maneuver phase, and $t_{\mathrm{f}}, v_{\mathrm{f}}$, and $h_{\mathrm{f}}$ represent the end time, final vertical velocity, and final altitude, respectively, of the hazard-avoidance maneuver phase. $t_{\max }, v_{\mathrm{f}}$, and $h_{\mathrm{f}}$ are designed to be $15 \mathrm{~s},-1.5 \mathrm{~m} / \mathrm{s}$, and $20 \mathrm{~m}$, respectively, for the Tianwen-1 lander. 
Let $a_{\mathrm{t} 2}=-a_{\mathrm{t} 1}$. Then, we obtain

$2 t_{\mathrm{max}} v_{\mathrm{m}}^{2}-4 h_{\mathrm{go}} v_{\mathrm{m}}+2 h_{\mathrm{go}}\left(v_{\mathrm{f}}+v_{0}\right)-\left(v_{\mathrm{f}}^{2}+v_{0}^{2}\right) t_{\max }=0$

According to Eq. (7), the maximum descent velocity $v_{\mathrm{m}}$ can be determined, and the magnitude of acceleration $a_{\mathrm{t}}$ can be computed as follows:

$$
a_{\mathrm{t}}=\frac{v_{\mathrm{f}}+v_{0}-2 v_{\mathrm{m}}}{t_{\max }}
$$

If $a_{\mathrm{t}}>a_{\mathrm{dmax}}$, we can obtain

$$
\left\{\begin{array}{l}
a_{\mathrm{t} 1}=-a_{\mathrm{t}} \\
a_{\mathrm{t} 2}=\frac{2 a_{\mathrm{t} 1} v_{\mathrm{f}}\left(a_{\mathrm{t} 1} t_{\mathrm{max}}+v_{0}\right)-a_{\mathrm{t} 1} v_{\mathrm{f}}^{2}-a_{\mathrm{t} 1} v_{0}^{2}-2 a_{\mathrm{t} 1}^{2} h_{\mathrm{go}}}{\left(a_{\mathrm{t} 1} t+v_{0}\right)^{2}-v_{0}^{2}-2 a_{\mathrm{t} 1} h_{\mathrm{go}}}
\end{array}\right.
$$

Otherwise, $a_{\mathrm{t} 1}=-a_{\mathrm{t}}$ and $a_{\mathrm{t} 2}=a_{\mathrm{t}}$.

According to the values of $a_{\mathrm{t} 1}$ and $a_{\mathrm{t} 2}$, the final maximum descent speed can be computed as follows:

$$
v_{\mathrm{m}}=\frac{a_{\mathrm{t} 1} a_{\mathrm{t} 2} t_{\mathrm{max}}+a_{\mathrm{t} 2} v_{0}-a_{\mathrm{t} 1} v_{\mathrm{f}}}{a_{\mathrm{t} 2}-a_{\mathrm{t} 1}}
$$

The switch time from uniform acceleration to uniform deceleration is given as

$$
t_{\mathrm{m}}=\frac{v_{\mathrm{m}}-v_{0}}{a_{\mathrm{t} 1}}
$$

Therefore, $h_{\mathrm{t}}, \dot{h}_{\mathrm{t}}$, and $\ddot{h}_{\mathrm{t}}$ are calculated as follows:

$$
\begin{aligned}
& \ddot{h}_{\mathrm{t}}= \begin{cases}a_{\mathrm{t} 1}, & t_{0} \leqslant t<t_{\mathrm{m}} \\
a_{\mathrm{t} 2}, & t_{0} \leqslant t<t_{\mathrm{f}}\end{cases} \\
& \dot{h}_{\mathrm{t}}= \begin{cases}v_{0}, & t=t_{0} \\
\dot{h}_{\mathrm{t}}+\ddot{h}_{\mathrm{t}} \cdot\left(t-t_{0}\right), & t_{0}<t\end{cases} \\
& h_{\mathrm{t}}= \begin{cases}h_{0}, & t=t_{0} \\
h_{\mathrm{t}}+\dot{h}_{\mathrm{t}} \cdot\left(t-t_{0}\right)+0.5 \cdot \ddot{h}_{\mathrm{t}} \cdot\left(t-t_{0}\right)^{2}, & t_{0}<t\end{cases}
\end{aligned}
$$

For the slow-descent phase, when the altitude is $\geqslant 10$ $\mathrm{m}$, the vertical command acceleration vector is defined as

$$
\boldsymbol{a}_{\mathrm{cmd}, \mathrm{ver}}=\left[-c_{2} \cdot\left(\dot{h}-\dot{h}_{\mathrm{t}}\right)-c_{3} \cdot\left(\ddot{h}-\ddot{h}_{\mathrm{t}}\right)+g_{\mathrm{m}}\right] \frac{\boldsymbol{r}}{\|\boldsymbol{r}\|}
$$

where $\dot{h}_{\mathrm{t}}=-1.5 \mathrm{~m} / \mathrm{s}$ and $\ddot{h}_{\mathrm{t}}=0 \mathrm{~m} / \mathrm{s}^{2}$.

When the altitude is $<10 \mathrm{~m}$, the vertical command acceleration vector is defined as

$$
\boldsymbol{a}_{\mathrm{cmd}, \mathrm{ver}}=\left(a_{\mathrm{cslw}}+g_{\mathrm{m}}\right) \frac{\boldsymbol{r}}{\|\boldsymbol{r}\|}
$$

where $a_{\mathrm{cslw}}=-0.05 \mathrm{~m} / \mathrm{s}^{2}$.

(2) Horizontal channel guidance and control

Inner loop control and outer loop control are designed for horizontal channel guidance and control. In the inner loop control, the horizontal maneuver is executed via the horizontal thrust from eight $250-\mathrm{N}$ thrusters. In the outer loop control, the horizontal-maneuver thrust from the main engine is obtained by performing an attitude maneuver. Generally, only inner loop control is used, and outer loop control can start only when the horizontal velocity is higher than $v_{\text {hmax }}$, which is designed to be 3,5 , and $3 \mathrm{~m} / \mathrm{s}$ for the hover and imaging phase, hazard-avoidance maneuver phase, and slow-descent phase, respectively, according to the normal horizontal velocity of each phase.

The inner loop control differs from the phase plane control of Chang'e lunar landers [12], and a horizontal maneuver control method based on the steepest-descent curve is designed to improve the rapidity of long-range maneuver control. In Fig. 6, the partition logic of horizontal maneuver control is described as follows.

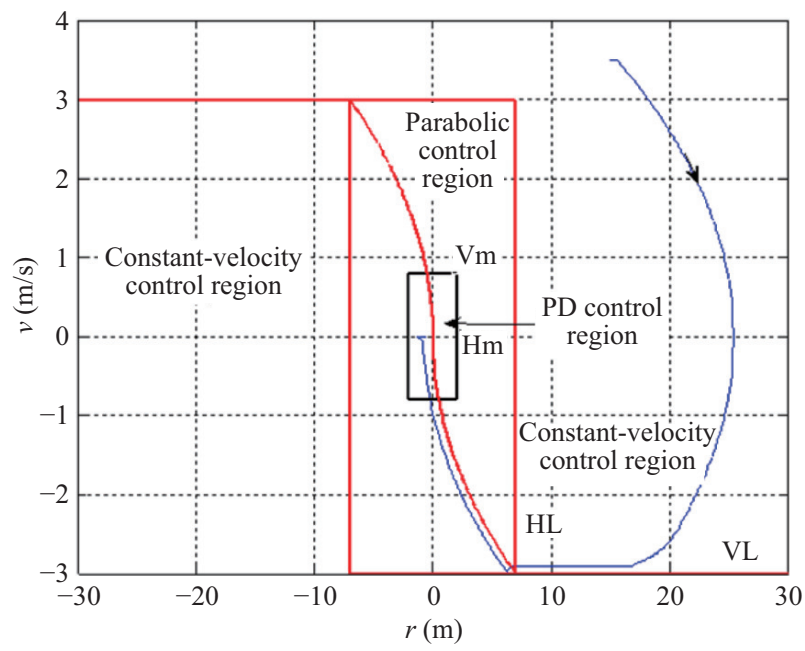

Fig. 6 Partition logic for horizontal maneuver control.

The outer zone is a constant-velocity control region, and when the absolute value of the position error is larger than HL $(7 \mathrm{~m})$, the control mode is adopted according to the constant target velocity VL $(3 \mathrm{~m} / \mathrm{s})$, and the control law is velocity-tracking proportional control $(\mathrm{P})$. The middle part is a parabolic control region, which is controlled according to the steepest-descent curve in the full spray mode. In the innermost proportional-differential (PD) control region, when the absolute value of the position error is smaller than $\mathrm{Hm}(2$ $\mathrm{m})$ and the velocity error is smaller than $\mathrm{Vm}(0.8 \mathrm{~m} / \mathrm{s})$, $\mathrm{PD}$ and pulse-width modulation (PWM) controlers are used for hazard-avoidance control.

In the outer loop control, the horizontal channel command acceleration is defined as 


$$
\boldsymbol{a}_{\mathrm{cmd}, \mathrm{hor}}=-d_{1} \cdot \boldsymbol{r}_{\mathrm{ICh}}-d_{2} \cdot \boldsymbol{v}_{\mathrm{ICh}}-d_{3} \cdot \boldsymbol{a}_{\mathrm{ICh}}
$$

where $\boldsymbol{r}_{\mathrm{ICh}}, \boldsymbol{v}_{\mathrm{ICh}}$, and $\boldsymbol{a}_{\mathrm{ICh}}$ represent the horizontal position, horizontal velocity, and horizontal acceleration, respectively, relative to the target landing site, and $d_{1}$, $d_{2}$, and $d_{3}$ are the PID control coefficients, which are designed to be $0.03,0.35$, and 0.01 , respectively.

If the outer loop control is started, the total command acceleration is computed as

$$
\boldsymbol{a}_{\mathrm{cmd}}=\boldsymbol{a}_{\mathrm{cmd}, \mathrm{ver}}+\boldsymbol{a}_{\mathrm{cmd}, \mathrm{hor}}
$$

The horizontal-maneuver thrust from the main engine is obtained by performing an attitude maneuver in the outer loop control. The attitude maneuver angle limiter is designed to prevent the attitude offset angle from becoming too large. Denoting the maximum attitude maneuver angle as $\theta_{\max }$, if $\left\|\boldsymbol{a}_{\mathrm{cmd} \text {,hor }}\right\| /\left\|\boldsymbol{a}_{\mathrm{cmd} \text {,ver }}\right\|>$ $\tan \theta_{\max }$, the horizontal channel command acceleration from Eq. (17) is limited by

$$
\boldsymbol{a}_{\mathrm{cmd}, \mathrm{hor}}=\tan \theta_{\max } \cdot\left\|\boldsymbol{a}_{\mathrm{cmd}, \mathrm{ver}}\right\| \cdot \frac{\boldsymbol{a}_{\mathrm{cmd}, \mathrm{hor}}}{\left\|\boldsymbol{a}_{\mathrm{cmd}, \text { hor }}\right\|}
$$

If the estimated horizontal velocity is $<10 \mathrm{~m} / \mathrm{s}, \theta_{\max }$ is designed to be $15^{\circ}$; otherwise, $\theta_{\max }=45^{\circ}$.

\subsection{Navigation algorithm}

The radar-updated inertial navigation strategy is employed for the PDL phase. The PDL-phase navigation framework is mainly inherited from the Chang'e-3 lander [13, 14, 17]. For each measurement, eight beams can be available, providing redundancy. Therefore, a multiple-beam fault detection, isolation, and recovery (FDIR) algorithm for landing radars, which is not contained in the navigation algorithm of the Chang'e-3 lander, was designed. Noting that the working beams for the PAR are not fixed, the velocity corrections for the case of coplanarity were also designed for Tianwen-1.
Once the velocimeter FDIR algorithm is implemented, the beams for velocity correction can be selected. For velocity correction, the first-order equation for the ground-relative velocity along each beam is established, and the velocity correction for each beam is updated using the Kalman filter, whose gain is approximated as a function of the velocity magnitude. The ground-relative velocity corrections are then fused to form a $3 \mathrm{D}$ velocity correction. The number of selected beams is not fixed for the correction, and multiple beams may be coplanar or almost coplanar. The fusion strategy depends on the number of selected beams. If only one beam is selected, only the velocity along this beam is corrected. In the case where two or more beams are selected, if they are coplanar or almost coplanar, a plane is constructed by two beams, and the velocity along the plane is corrected using the least-squares method; otherwise, a 3D velocity correction is performed. Once the safe landing site is determined by the hazard identification and safe landing site selection algorithm during the hazard-avoidance and CBP-avoidance maneuvers, navigation relative to the landing site begins. A flowchart of the PDL navigation algorithm is shown in Fig. 7. Details regarding the algorithm were presented in previous works [18].

\subsection{Attitude-control algorithm}

Owing to the uncertainties of the parachute descent phase, the state dispersion from the nominal values may be large at the beginning of the PDL phase. Therefore, the attitude controller should be robust. For example, the attitude controller in the powered-deceleration mode should track the guidance command with a maximum angular rate of $15\left(^{\circ}\right) / \mathrm{s}$. Rapid attitude tracking may suffer from large disturbances. The attitude phase

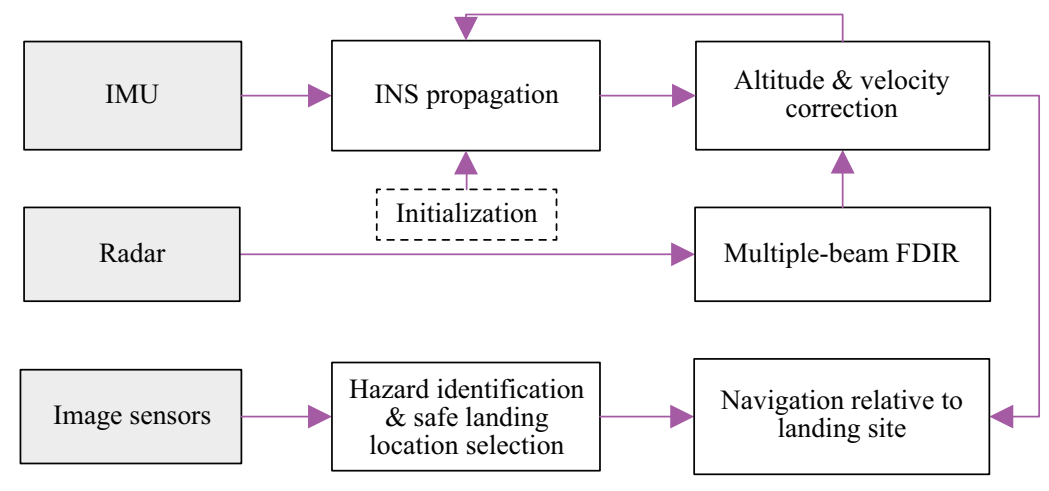

Fig. 7 Flowchart of the PDL navigation algorithm. 
plane is partitioned into four regions [19]: a PID control region, nominal rate control region, rate limiter region, and parabolic target rate control region. In the nominal rate and parabolic target rate control regions, proportional-integral (PI)+PWM controllers are used to track the desired rate. In the rate limiter region, when the angular rate exceeds the threshold, the available thrusters drive the angular rate to the set range. In the PID control region, the traditional PID + PWM controller [20] is selected to track the desired attitude angle and angular rate. The four attitude-control regions are applied according to the value range of the angle errors between the lander's attitude and the commanded attitude.

Several additional strategies are designed. First, observers are designed for online disturbance identification and compensation. Second, the commanded thrust direction is decoupled from the attitude and sent directly to the pitch and yaw channels, which allows the tracking of the commanded thrust direction to be as fast as possible. Third, an attitude controller based on multiple-level thruster switching logic is designed according to the attitude error. Finally, an FDIR algorithm is designed for Tianwen-1 to identify fault thrusts and rearrange the remaining thrusts, which makes the controller more robust if no thruster can be opened. Details regarding the attitude-control algorithm were presented in a previous work [21].

\subsection{Hazard identification and safe landing site selection algorithm}

\subsubsection{Hazard identification and safe landing site} selection algorithm based on optical images

The hazard identification and safe landing site selection algorithm based on optical images is inherited from the Chang'e-3 lander [12, 14] and optimized and improved according to the surface environment and landing characteristics of Mars. It is summarized as follows.

(i) Hazard identification based on the surface texture of Mars: the texture algorithm is used to calculate the roughness of the table, determine the positions of the hazards, and calculate the safety area. (ii) Safety probability calculation and sorting: the safety probabilities of different areas are obtained according to the texture hazard results; five safety areas are selected; and the area with the highest safety probability is output (if a plurality of areas with the highest safety probability exists, the first area is output).

4.4.2 Hazard identification and safe landing site selection algorithm based on $3 D$ terrain data

The hazard identification and safe landing site selection algorithm based on 3D terrain data is inherited from the Chang'e-3 lander [12, 14] and optimized and improved according to the surface environment and landing characteristics of Mars. It is summarized as follows.

(i) Data normalization: the calculation, extraction, and resampling of the $256 \times 256$ array data are performed. (ii) Region normalization: terrain region division is performed via DEM data gridding. (iii) Hazard identification: slope fitting, elevation judgment, and safety probability calculations are performed. (iv) Calculation of safe landing site: areas are sorted and output according to their safety probabilities.

\subsubsection{Hazard identification and safe landing site} selection algorithm based on binocular vision

A hazard identification and safe landing site selection algorithm based on binocular vision is designed. According to the principle of stereo imaging, the slope and elevation information of the landing area is obtained and used as a backup for hazard identification. The algorithm is summarized as follows. (i) Texture hazard identification: local texture assessment for a single image is used to determine the hazard area and first level of security. (ii) Computation of disparity map: a dense binocular matching method is used for binocular matching to obtain a disparity map with a low processing rate and to obtain $256 \times 2563 \mathrm{D}$ elevation data. With the $3 \mathrm{D}$ elevation data, the processing is identical to that of the aforementioned algorithm for autonomous hazard identification and landing area selection based on 3D terrain data.

\section{$5 \quad$ PDL GNC system flight results}

In this section, the actual flight results for the Tianwen-1 PDL process are presented. The desired performance of the Tianwen-1 mission GNC system was discussed in Ref. [22]. The actual PDL flight process is described as follows according to the delay telemetry data. At a navigated altitude of $1.368 \mathrm{~km}$ and navigated velocity of $57.65 \mathrm{~m} / \mathrm{s}$, the CBP was separated, implying the beginning of the PDL phase, which lasted $90 \mathrm{~s}$. The O-HAS obtained optical images of the predefined 
landing area after CBP separation. The optical images were processed, and a safe landing site was identified according to the constraints of both CBP avoidance and coarse hazard avoidance. Approximately $1 \mathrm{~s}$ after CBP separation, the MLE was ignited, the landing rover's velocity was reduced further, and a $\mathrm{CBP}$ and coarse hazard-avoidance maneuver was performed. When the landing rover's altitude was reduced to approximately 100 $\mathrm{m}$, the GNC switched to the hover and imaging mode. In this mode, the M-HAS obtained 3D images of the Mars surface and determined the final safe landing site. The GNC then switched to the hazard-avoidance maneuver mode. Horizontal thrusters were used to perform precise hazard-avoidance maneuvers, and the landing rover reached a position $20 \mathrm{~m}$ above the final safe landing site. When the landing rover's altitude was $<20 \mathrm{~m}$, the GNC switched to the slow-descent mode. The landing rover descended at a constant vertical velocity of approximately $1.5 \mathrm{~m} / \mathrm{s}$ and continued to reduce the horizontal velocity. The landing rover landed on Mars softly with a stable vertical attitude. The touchdown horizontal velocity was $<0.16 \mathrm{~m} / \mathrm{s}$, and the attitude error was $<0.1^{\circ}$.

The actual PDL flight attitude, altitude, trajectories, and images from the delay telemetry data are presented in Figs. 8-17. As shown in Fig. 12, the selected safe landing site can simultaneously satisfy the requirements of CBP and coarse hazard avoidance. Figure 13 shows the trajectory of the landing rover approaching the selected safe landing site during the powered-deceleration mode. The actual distance between the CBP and the landing site was $>350 \mathrm{~m}$ according to the landing-area image (Fig. 14) taken by the Tianwen-1 orbiter. Figure 15 shows

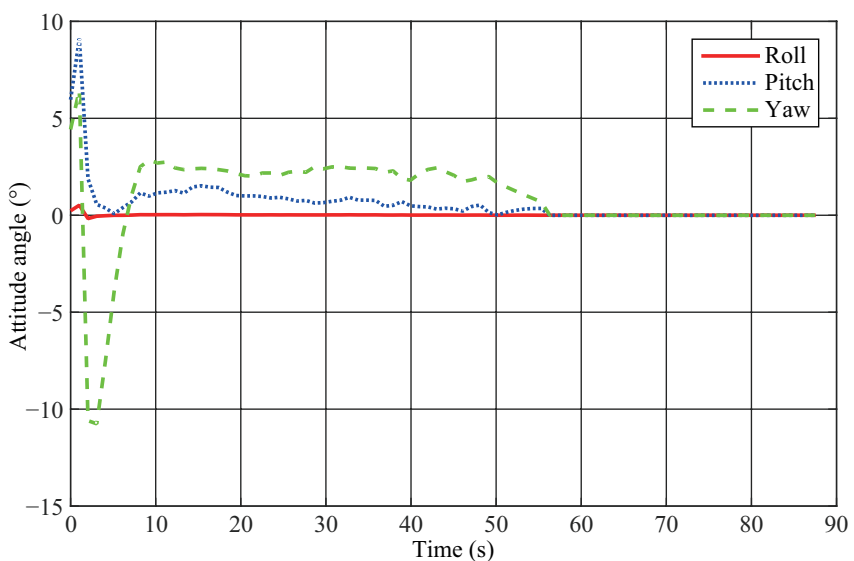

Fig. 8 Attitude angles between the landing-rover body and local up-south-east during the PDL phase.

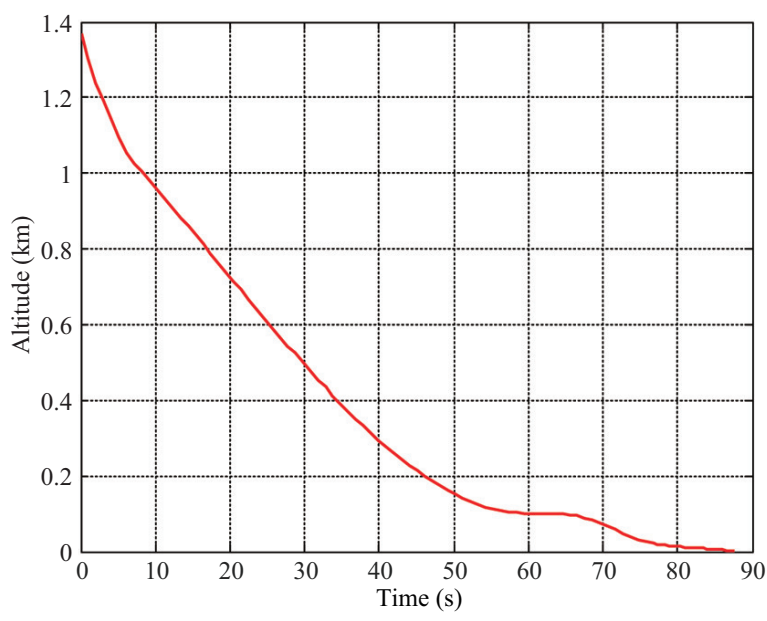

Fig. 9 Altitude relative to the Mars surface during the PDL phase.

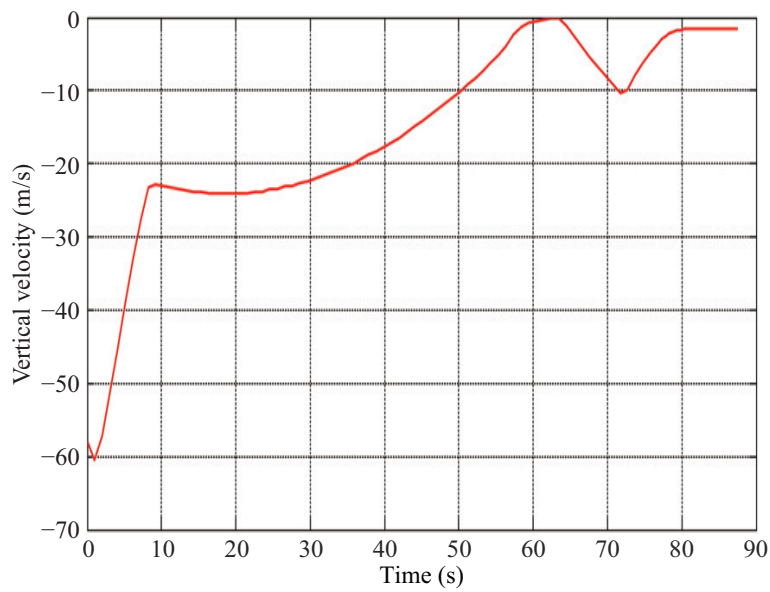

Fig. 10 Vertical velocity relative to the Mars surface during the PDL phase.

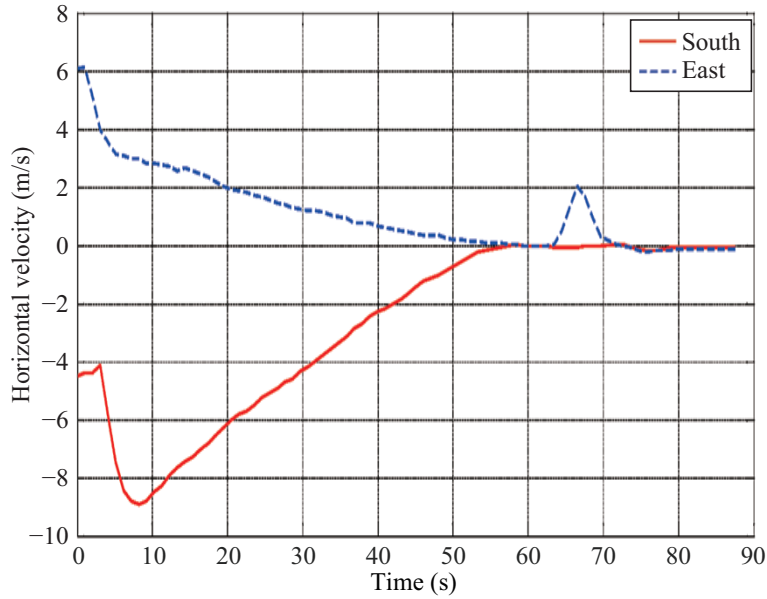

Fig. 11 Horizontal velocity relative to the Mars surface during the PDL phase.

the selected safe landing site based on a 3D terrain map of the landing area. Figure 16 shows the trajectory of the

\section{(10) $\operatorname{TSINGHUA}$ UNIVRSIYYRESA Springer}




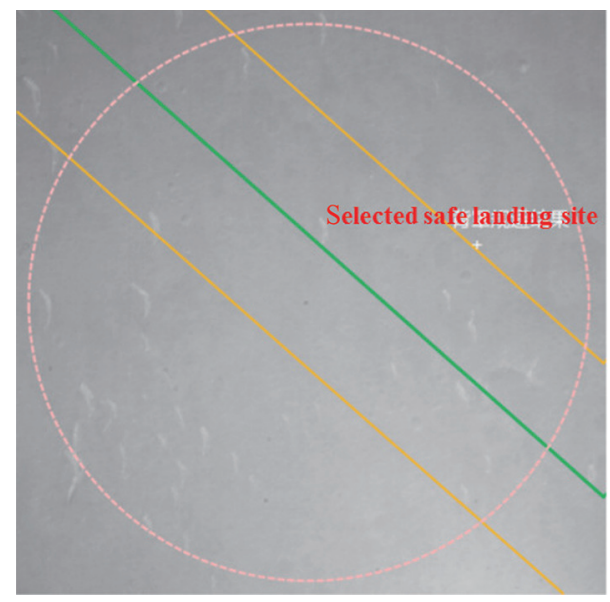

Fig. 12 CBP- and hazard-avoidance image showing the selected safe landing site.

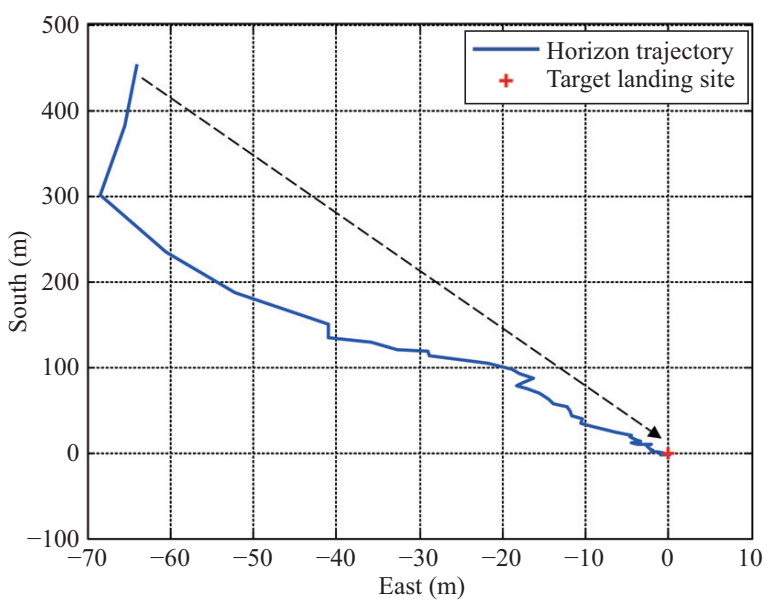

Fig. 13 Flight trajectory relative to the landing site for CBP and hazard avoidance.

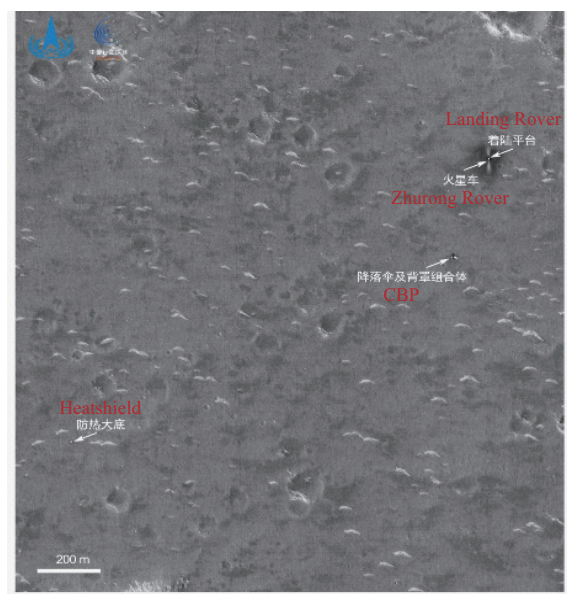

Fig. 14 Real position relationship between the CBP touchdown point and the landing site.

landing rover approaching the selected safe landing site during the hazard-avoidance maneuver mode. The real

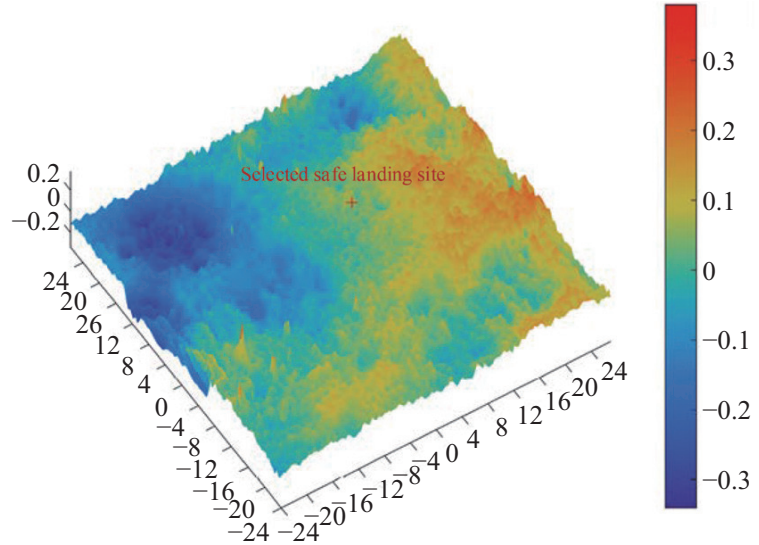

Fig. 15 3D terrain map of the landing area and selected safe landing site.

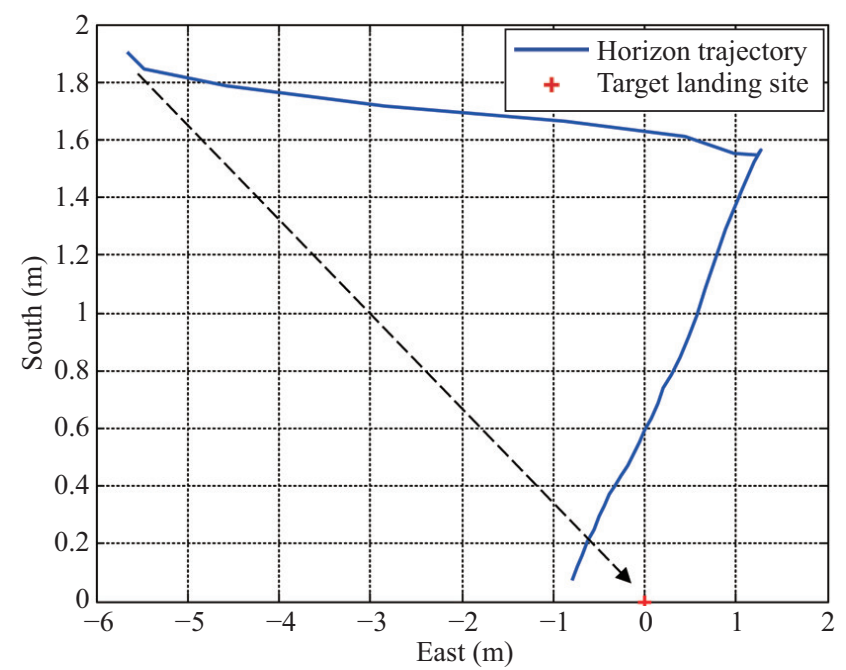

Fig. 16 Flight trajectory relative to the landing site for precise hazard avoidance.

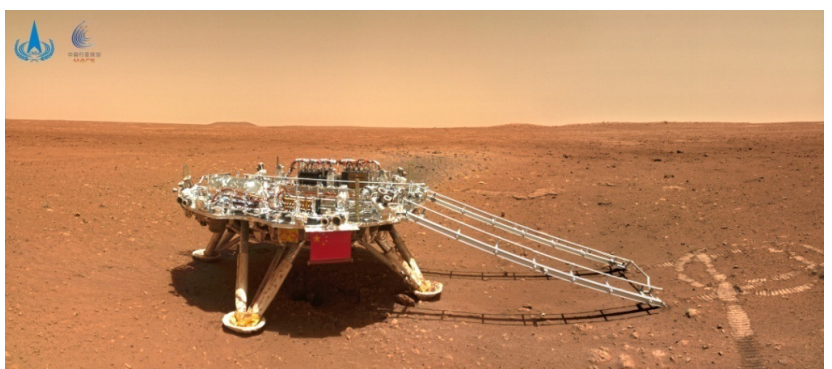

Fig. 17 Real landing-site terrain and the Tianwen-1 landing rover.

landing-site terrain of the Tianwen-1 landing rover was safe according to the landing-area image (Fig. 17) taken by the Zhurong rover. The results indicate that the CBP and hazards were successfully avoided and that the safety of both the landing-area terrain and the touchdown state were ensured in the Tianwen-1 mission.

(i) TSINGHUA 


\section{Conclusions}

This paper presented the PDL GNC system design and flight results for the Tianwen-1 mission. The analysis indicated that the trajectory maneuver to avoid collision with the CBP and large-scale hazards was simultaneously and successfully performed, the precise hazard-avoidance maneuver and velocity and altitude control were implemented, and the landing rover was placed gently and safely on the surface of Mars. The execution of the PDL GNC resulted in Tianwen-1's successful touchdown in the morning of 15 May 2021. Thus, Tianwen-1 achieved a historic, first autonomous landing with simultaneous hazard and CBP avoidance.

\section{Acknowledgements}

This work was supported by the National Natural Science Foundation of China (Grant Nos. 61673057 and 61803028) and the Civil Aerospace Advance Research Project.

\section{Conflict of interest}

The authors declare that there is no conflict of interest.

\section{References}

[1] Wang, D., Huang, X., Guan, Y. GNC system scheme for lunar soft landing spacecraft. Advances in Space Research, 2008, 42(2): 379-385.

[2] Busnardo, D. M., Aitken, M. L., Tolson, R. H., Pierrottet, D., Amzajerdian, F. LIDAR-aided inertial navigation with extended Kalman filtering for pinpoint landing over rough terrain. In: Proceedings of the 49th AIAA Aerospace Science Meeting including the New Horizons Forum And Aerospace Exposition, 2011: AIAA 2011-428.

[3] Xu, C., Wang, D., Huang, X. Landmark-based autonomous navigation for pinpoint planetary landing. Advance in Space Research, 2016, 58(11): 2313-2327.

[4] Xu, C., Wang, D., Huang, X. Autonomous navigation based on sequential images for planetary landing in unknown environments. Journal of Guidance, Control, and Dynamics, 2017, 40(10): 2587-2602.

[5] Owens, C., Macdonald, K., Hardy, J., Lindsay, R., Redfield, M., Bloom, M., Bailey, E., Cheng, Y., Clouse, D., Villalpando, C. Y., et al. Development of a signature-based terrain relative navigation system for precision landing. In: Proceedings of the AIAA Scitech 2021 Forum, 2021: AIAA 2021-0376.

[6] Fahroo, F., Ross, I. M. Trajectory optimization by indirect spectral collocation methods. In: Proceedings of the Astrodynamics Specialist Conference, 2000: AIAA 2000-4028.

[7] Acikmese, B., Carson, J. M., Blackmore, L. Lossless convexification of nonconvex control bound and pointing constraints of the soft landing optimal control problem. IEEE Transactions on Control Systems Technology, 2013, 21(6): 2104-2113.

[8] Ploen, S., Acikmese, B., Wolf, A. A comparison of powered descent guidance laws for mars pinpoint landing. In: Proceedings of the AIAA/AAS Astrodynamics Specialist Conference and Exhibit, 2006: AIAA 2006-6676.

[9] Martella, P., Buonocore, M., Canuto, E., MolanoJimenez, A., Drai, R., Lorenzoni, L. Design and verification of the GNC for the European ExoMars EDL deomonstrator. In: Proceedings of the AIAA Guidance, Navigation, and Control Conference, 2011: AIAA 2011-6341.

[10] Sell, S. W., Davis, J. L., Martin, A. M. S., Serricchio, F. Powered fight design and performance summary for the Mars Science Laboratory mission. Journal of Spacecraft and Rockets, 2014, 51(4): 1197-1207.

[11] San Martin, A. M., Lee, S. W., Wong, E. C. The development of the MSL guidance, navigation, and control system for entry, descent, and landing. In: Proceedings of the AAS/AIAA Space Flight Mechanics Meeting, 2013: 529-546.

[12] Zhang, H. H., Liang, J., Huang, X. Y., Zhao, Y., Wang, L., Guan, Y. F., Cheng, M., Li, J., Wang, P. J., Yu, J., et al. Autonomous hazard avoidance control for Chang'e-3 soft landing. SCIENTIA SINICA Technologica, 2014, 44(6): 559-568. (in Chinese)

[13] Huang, X. Y., Zhang, H. H., Wang, D. Y., Li, J., Guan, Y. F., Wang, P. J. Autonomous navigation and guidance for Chang'e-3 soft landing. Journal of Deep Space Exploration, 2014, 1(1): 52-59. (in Chinese)

[14] Wang, D. Y., Li, J., Huang, X. Y., Zhang, H. H. A pinpoint autonomous navigation and hazard avoidance method for lunar soft landing. Journal of Deep Space Exploration, 2014, 1(1): 44-51. (in Chinese)

[15] Zhang, H. H., Guang, Y. F., Cheng, M., Li, J., Yu, P., Zhang, X. W., Wang, H. Q., Yang, W., Wang, Z. W., Yu, J., et al. Guidance navigation and control of Chang'e-4 lander. SCIENTIA SINICA Technologica, 2019, 49(12): 1418-1428. (in Chinese)

[16] Yu, P., Zhang, H. H., Li, J., Guan, Y. F., Wang, L., Zhao, Y., Chen, Y., Yang, W., Yu, J., Wang, H. Q., et al. Design and implementation of GNC system of lander and ascender module of Chang'e-5 spacecraft. SCIENTIA SINICA Technologica, 2021, 51(7): 763-777. (in Chinese) 
[17] Zhang, H. H., Li, J., Guan, Y. F., Huang, X. Y. Autonomous navigation for powered descent phase of Chang'e-3 lunar lander. Control Theory $\&$ Applications, 2014, 31(12): 1686-1694. (in Chinese)

[18] Huang, X. Y., Li, M. D., Wang, X. L., Hu, J. C., Zhao, Y., Guo, M. W., Xu, C., Liu, W. W., Wang, Y. P., Hao, C., Xu, L. J. The Tianwen-1 guidance, navigation, and control for Mars entry, descent, and landing. Space: Science \& Technology, 2021, 2021: 9846185.

[19] Zhang, H. H., Guan, Y. F., Hu, J. C., Wang, Z. G. A novel attitude control strategy based on quaternion partition. Acta Automatica Sinica, 2015, 41(7): 1341-1349. (in Chinese)

[20] Wang, Z., Li, T. S., Wang, D. Y. Attitude control during lunar satellite orbit maneuver. Aerospace Control, 2005, 23(1): 11-14. (in Chinese)

[21] Hu, J. C., Huang, X. Y., Li, M. D., Guo, M. W., Xu, C., Zhao, Y., Liu, W. W., Wang, X. L. Entry vehicle control system design for the Tianwen-1 mission. Astrodynamics, 2022, 6(1): 27-37.

[22] Xu, C., Huang, X. Y., Guo, M. W., Li, M. D., Hu, J. C., Wang, X. L. End-to-end Mars entry, descent, and landing modeling and simulations for the Tianwen-1 guidance, navigation, and control system. Astrodynamics, 2022, 6(1): 53-67.

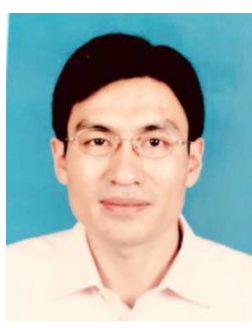

Xiangyu Huang received his B.S. degree and Ph.D. degree in aerospace engineering from Harbin Institute of Technology, Harbin, China, in 1999 and 2005, respectively. Since August 2005, he has been with Science and Technology on Space Intelligent Control Laboratory of Beijing Institute of Control Engineering (BICE), where he is currently a professor and senior research specialist. He was a GNC engineer of the Chang'e-3 lunar landing mission, and his current research area is the GNC design for planetary landing missions. He won one National Technological Innovation Prize (second class) and three ministerial level prizes (first class). E-mail: huangxyhit@sina.com.

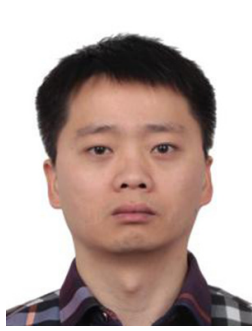

Chao $\mathbf{X u}$ received his B.S. degree from Beihang University, Beijing, China, in 2010, and received his M.E. and Ph.D. degrees in guidance, navigation, and control from China Academy of Space Technology (CAST), Beijing, China, in 2013 and 2017, respectively. $\mathrm{He}$ is currently working as an engineer at Beijing Institute of Control Engineering (BICE), Beijing, China. His research interests include autonomous navigation, vision-aided navigation, and simultaneous localization and mapping. E-mail: xc_1987@126.com.

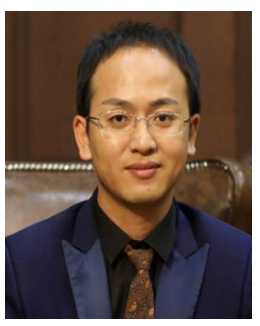

Jinchang Hu received his B.S. degree from Beihang University, M.E. degree from Tsinghua University, and Ph.D. degree from Beijing Institute of Control Engineering. He is now a senior engineer of Beijing Institute of Control Engineering. His main research interest lies in attitude and orbit control of spacecraft. E-mail: hujinchang@tsinghua.org.cn.

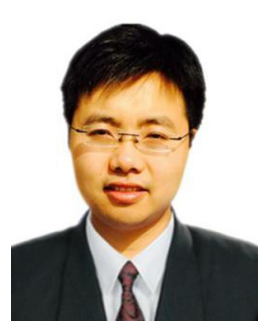

Maodeng Li received his B.S. and Ph.D. degrees in aerospace engineering from Harbin Institute of Technology, Harbin, China, in 2006 and 2011, respectively. From September 2011 to August 2013, he was a postdoctoral research associate in Beijing Institute of Control Engineering (BICE). Since August 2013, he has been with Science and Technology on Space Intelligent Control Laboratory of BICE as a senior engineer. His current research areas include spacecraft autonomous navigation and GNC design for planetary landing missions. E-mail: mdeng1985@gmail.com.

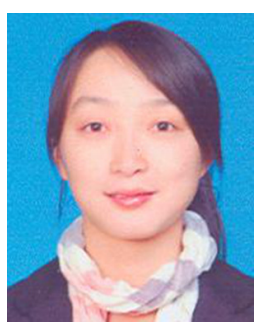

Minwen Guo received her Ph.D. degree in navigation, guidance, and control from China Academy of Space Technology in 2014. Then she worked as an engineer in Beijing Institute of Control Engineering. From 2016 to 2021, she participated in the Mars Tianwen-1 development and was engaged in the design of Martian atmosphere entry guidance law. In 2019 she was named senior engineer. Currently, her research interests are spacecraft entry guidance and trajectory optimization. E-mail: mwguo8500@163.com.

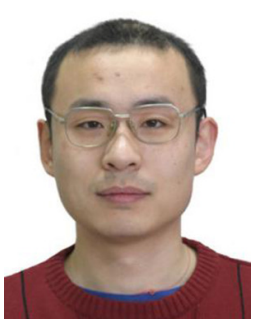

Xiaolei Wang is now a professor of Beijing Institute of Control Engineering. His main research interest lies in spacecraft GNC system design. 


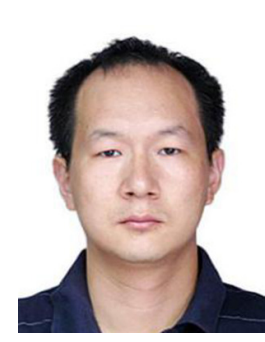

Yu Zhao is now a professor of Beijing Institute of Control Engineering. His main research interest is spacecraft GNC system design. E-mail: 1336618606@163.com.

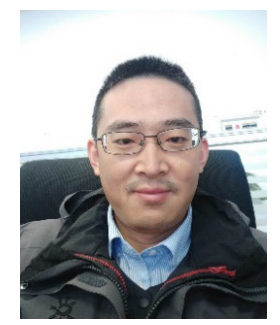

Baocheng Hua received his B.S. degree from North University of Technology in 2005 and his Ph.D. degree from Beijing University of Aeronautics and Astronautics in 2011. He is currently a senior engineer at the Beijing Institute of Control Engineering. His main research interests are machine vision, optical navigation, deep space exploration, etc. E-mail: 85340287@qq.com.

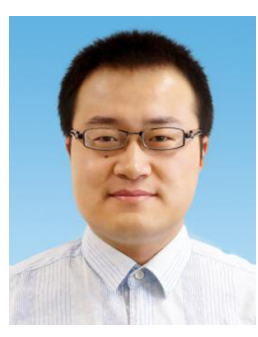

Yunpeng Wang received his B.S. degree in information and computing science from Shandong University, China, in July 2010, and his Ph.D. degree in control theory and control engineering from the Institute of Automation, Chinese Academy of Sciences, in July 2015. He is currently a senior engineer at the Beijing Institute of Control Engineering. His current research interests include the spacecraft control and multi-agent system. E-mail: yunpeng_wang@foxmail.com.

Open Access This article is licensed under a Creative Commons Attribution 4.0 International License, which permits use, sharing, adaptation, distribution and reproduction in any medium or format, as long as you give appropriate credit to the original author(s) and the source, provide a link to the Creative Commons licence, and indicate if changes were made.

The images or other third party material in this article are included in the article's Creative Commons licence, unless indicated otherwise in a credit line to the material. If material is not included in the article's Creative Commons licence and your intended use is not permitted by statutory regulation or exceeds the permitted use, you will need to obtain permission directly from the copyright holder.

To view a copy of this licence, visit http://creativecomm ons.org/licenses/by/4.0/. 